\title{
Dapsone syndrome following treatment for paucibacillary leprosy manifesting with prolonged exfoliative dermatitis and granulomatous hepatitis
}

\author{
M C Shivanthan ${ }^{1}$, K Satgurunathan ${ }^{1}$ \\ Journal of the Ceylon College of Physicians, 2011, 42, 35-36
}

\section{Introduction}

Dapsone hypersensitivity syndrome is a well established entity and has been reported in literature since the 1950s. A unique case of dapsone syndrome which had a very prolonged course lasting eight months is reported with no similar reports in literature. With the wide use of dapsone in many medical conditions, clinicians should be aware that dapsone can cause a prolonged clinical syndrome of dapsone hypersensitivity which might mimic other sinister conditions.

Key words: dapsone hypersensitivity syndrome, granulomatous hepatitis, exfoliative dermatitis, leprosy, dapsone

\section{Case report}

A 40-year old female presented with a 3 month history of fever, nausea, right hypochondrial pain, generalised pruritic skin rash and frothy urine, after the complete withdrawal of antileprosy treatment which was commenced 3 weeks preceding the onset of her symptoms, after being diagnosed with of paucibacillary leprosy. Anti leprosy treatment comprised of $100 \mathrm{mg}$ of dapsone daily with monthly rifampicin, and only one dose of the latter had been taken prior to discontinuation. Low doses of prednisolone had been repeatedly prescribed to treat her symptoms which were attributed to dapsone syndrome however with poor response. She had never taken any herbal treatment or other forms of native treatment. Exposure to heavy metals was excluded. She had not been on any other medications preceding her symptoms. Examination revealed a diffuse maculopapular exfoliative skin rash with alopecia, dependant oedema and fever. Jaundice, pallor and lymphadenopathy were absent. The mucosae were normal. Cardiovascular respiratory and neurological system examination findings were unremarkable. A $2 \mathrm{~cm}$ smooth, regular, non tender hepatomegaly was detected.

\footnotetext{
${ }^{1}$ Dermatology Unit I, National Hospital of Sri Lanka, Colombo.
}

Laboratory investigations at this stage revealed $\mathrm{Hb} 14.8 \mathrm{~g} / \mathrm{dL}$, WBC 18700/mm ${ }^{3}$, neutrophils 57\%, lymphocytes $16 \%$, eosinophils $24 \%$. Blood picture showed eosinophilia. Liver function tests revealed total bilirubin $15 \mathrm{umol} / \mathrm{l}$ with a direct fraction of $8 \mathrm{umol} / \mathrm{l}$, AST $400 \mathrm{U} / \mathrm{L}$, ALT $877 \mathrm{U} / \mathrm{L}$, alkaline phosphatase 131 $\mathrm{U} / \mathrm{L}$, serum albumin $32 \mathrm{~g} / \mathrm{l}$ and a prothrombin time of $13 \mathrm{~s}$ against a control of $12 \mathrm{~s}$. Serological tests for hepatitis A virus (anti-HAV IgM), hepatitis B virus (HBsAg), hepatitis $C$ virus (anti-HCV) were negative. Blood for LE cells and serum ANA were negative. Ultrasound abdomen, showed mild uniform enlargement of liver with normal echo-texture, and no evidence of portal hypertension or biliary dilatation. Urine full report showed 25-30 pus cells per high power field. Serum creatinine was $67 \mathrm{umol} / \mathrm{l}$. Blood and urine cultures were sterile. The skin biopsy was concluded as being compatible with exfoliative dermatitis. Liver biopsy showed intact parenchymal architecture. Portal tracts revealed mononuclear cell infiltration with formation of vague granulomas of epithelioid histiocytes and admixed eosinophils. There was no bile duct abnormalities or interface hepatitis. Hepatic parenchyma demonstrated micro and macro vesicular steatosis without any fibrosis. The biopsy was concluded as being highly suggestive of drug induced granulomatous hepatitis.

The patient was treated with antipyretics, and intravenous hydrocortisone followed by high dose oral prednisolone (1 $\mathrm{mg} / \mathrm{kg} / \mathrm{d})$, topical steroids, emollients. Antihistamines were prescribed for pruritus. Antimicrobial cover was commenced until the negative cultures were received. Fever resolved within 4 days of commencement of high dose steroids and liver enzymes showed a declining trend. Although there was clinical and biochemical improvement of her condition with steroids she relapsed frequently during the initial 3 months whenever a steroid taper was attempted, and therefore she was treated with a prolonged course of slowly tapering prednisolone for almost 8 months with bone protection. Her recovery was complicated by transient steroid induced diabetes mellitus which was controlled with dietary measures and medication. But this subsequently resolved with reducing doses of steroids. She completely recovered and is currently 
not on any medications. On review at 9 months she was free of dermatological, hepatic or other systemic complaints or signs, and her blood counts, liver enzymes, blood sugars and urine full report parameters were all within normal limits.

\section{Discussion}

Dapsone is one of the mainstay drugs in the treatment of leprosy ${ }^{1}$. It is also used in many other conditions $^{2}$. 'Dapsone syndrome' is a generalized hypersensitivity reaction that occurs in patients on dapsone therapy ${ }^{3}$. The cardinal features are fever, exfoliative dermatitis, lymphadenopathy, Iymphocytosis, methemoglobinemia, hemolytic anemia and hepatotoxicity. Dapsone syndrome is a type of drug rash with eosinophilia and systemic symptoms (DRESS) syndrome 4 .

Hyperbilirubinemia when present in dapsone syndrome occurs due to haemolysis ${ }^{5}$ and also from hepatocellular and cholestatic liver injury ${ }^{6}$. Liver biopsy shows either hepatitis, cholestasis or non caseous granulomas ${ }^{7}$ though it is usually not performed routinely.

The single dose of rifampicin which the patient had received is unlikely to have been the causative agent. Exfoliative dermatitis is more common with dapsone than other implicated agents.

Our case had the typical features of dapsone syndrome without an apparent alternative aetiology for the presentation. The symptoms appeared within a month of starting of dapsone with sterile pyuria from renal involvement ${ }^{8}$.

Drug induced liver granulomas are seen with numerous pharmacologic agents ${ }^{9}$. The classical histological feature of drug induced liver damage firmly supported the diagnosis after exclusion of other likely causatives including herbal medications.
Treatment is withdrawal of dapsone and high dose steroids. Dapsone persists in the body for well over 35 days therefore gluocorticoids are best tapered slowly ${ }^{10}$. However, this patient had an unusually prolonged dapsone hypersensitivity syndrome persisting for 8 months following the withdrawal of dapsone. Literature search failed to reveal documented precedence of a similar prolonged course. The authors conclude that clinicians need to be aware of a prolonged clinical course in dapsone syndrome.

\section{References}

1. Yawalkar SJ, McDougall AC, Languillon J, Ghosh S, Hajra SK, Opromolla DV, Tonello CJ. Once-monthly rifampicin plus daily dapsone in initial treatment of lepromatous leprosy. Lancet 1982; 1: 1199-202.

2. Wozel VE. Innovative use of dapsone. Dermatol Clin 2010; 28: 599-610.

3. Agrawal S, Agarwalla A. Dapsone hypersensitivity syndrome: a clinico-epidemiological review. J Dermatol 2005; 32: 883-9.

4. Millikan LE, Harrell ER. Drug reactions to the sulfones. Arch Dermatol 1970; 102: 220-4.

5. DeGowin RL. A review of therapeutic and hemolytic effects of dapsone. Arch Intern Med 1967; 120: 242-8.

6. Johnson DA, Cattau EL Jr, Kuritsky JN, Zimmerman HJ. Liver involvement in the sulfone syndrome. Arch Intern Med 1986; 146: 875-7.

7. Lons T, Richardet JP, Machayekhi JP, Dalbergue B, Trinchet JC. Granulomatous hepatitis caused by dapsone. Gastroenterol Clin Biol 1992; 16: 293.

8. Zhu YI, Stiller MJ. Dapsone and sulfones in dermatology: overview and update. J Am Acad Dermatol 2001; 45: 42034.

9. Zimmerman $\mathrm{HJ}$, Lewis $\mathrm{JH}$. Chemical and toxin-induced hepatotoxicity. Gastroenterol Clin North Am 1995; 24: 102745.

10. Kosseifi SG, Guha B, Nassour DN, Chi DS, Krishnaswamy G. The dapsone hypersensitivity syndrome revisited: a potentially fatal multisystem disorder with prominent hepatopulmonary manifestations. J Occup Med Toxicol 2006; 1: 9. 